\title{
TLR4 links innate immunity and fatty acid- induced insulin resistance
}

\author{
Hang Shi, Maia V. Kokoeva, Karen Inouye, Iphigenia Tzameli, Huali Yin, and Jeffrey S. Flier \\ Division of Endocrinology, Department of Medicine, Beth Israel Deaconess Medical Center, and Harvard Medical School, Boston, Massachusetts, USA.
}

\begin{abstract}
TLR4 is the receptor for LPS and plays a critical role in innate immunity. Stimulation of TLR4 activates proinflammatory pathways and induces cytokine expression in a variety of cell types. Inflammatory pathways are activated in tissues of obese animals and humans and play an important role in obesity-associated insulin resistance. Here we show that nutritional fatty acids, whose circulating levels are often increased in obesity, activate TLR4 signaling in adipocytes and macrophages and that the capacity of fatty acids to induce inflammatory signaling in adipose cells or tissue and macrophages is blunted in the absence of TLR4. Moreover, mice lacking TLR4 are substantially protected from the ability of systemic lipid infusion to (a) suppress insulin signaling in muscle and (b) reduce insulin-mediated changes in systemic glucose metabolism. Finally, female C57BL/6 mice lacking TLR4 have increased obesity but are partially protected against high fat diet-induced insulin resistance, possibly due to reduced inflammatory gene expression in liver and fat. Taken together, these data suggest that TLR4 is a molecular link among nutrition, lipids, and inflammation and that the innate immune system participates in the regulation of energy balance and insulin resistance in response to changes in the nutritional environment.
\end{abstract}

\section{Introduction}

Obesity is the most important identified factor contributing to insulin resistance $(1,2)$. The physiological mechanisms linking obesity to insulin resistance have received intense investigation and include altered production of various adipocyte-derived molecules, among which are the adipokines adiponectin and resistin, proinflammatory cytokines such as TNF- $\alpha$ and IL-6, RBP4, and FFAs themselves (1-7). It is increasingly recognized that obesity is characterized by chronic activation of inflammatory pathways and that inflammatory signaling pathways in obesity are causally linked to insulin resistance $(1,2,7)$. However, the fundamental mechanisms responsible for activating inflammatory pathways in obesity are poorly understood. FFAs, levels of which are elevated in obesity, due to increased release from expanded adipose tissue, have been hypothesized to cause insulin resistance. Indeed, FFA infusion in vivo has been shown to impair the ability of insulin to suppress hepatic glucose production and to stimulate glucose uptake into skeletal muscle $(8-10)$, which in turn leads to insulin resistance. Potential intracellular mechanisms whereby FFAs cause insulin resistance have been explored, and a role for several inflammatory signaling networks has emerged. For instance, intracellular kinases linked to inflammatory signaling, including PKC- $\theta$, IKK $\alpha$, and JNK appear to play roles in fatty acid-induced insulin resistance, since $P K C-\theta-, I K K \alpha$ - and $J N K$-knockout mice are substantially protected from FFA-induced insulin resistance (11-14). However, while fatty acid-induced insulin resistance appears to involve activation of intracellular inflammatory signaling pathways, the primary molecular mechanisms whereby FFAs activate these signaling networks have so far remained obscure.

Nonstandard abbreviations used: ChIP, chromatin immunoprecipitation DIO, diet-induced obese; IRS-1, insulin receptor substrate-1; MCP-1, monocyte chemoattractant protein-1; MyD88, myeloid differentiation factor 88

Conflict of interest: The authors have declared that no conflict of interest exists. Citation for this article: J. Clin. Invest. 116:3015-3025 (2006). doi:10.1172/JCI28898.
TLRs are a family of pattern-recognition receptors that play a critical role in the innate immune system by activating proinflammatory signaling pathways in response to microbial pathogens (15). TLR4, the best-characterized TLR, binds to LPS of gram-negative bacterial cell walls (15). Upon binding of LPS to TLR4 and its coreceptors CD14 and MD-2, the adaptor protein myeloid differentiation factor 88 (MyD88) is recruited to the Toll/IL-1 receptor (TIR) domain of the receptor (16). Interaction of the TIR domain of TLR4 and MyD88 triggers a downstream signaling cascade, leading to activation of the NF- $\kappa$ B pathway, which then activates the transcription of many proinflammatory genes that encode proinflammatory molecules including cytokines, chemokines, and other effectors of the innate immune response (16). In light of data indicating a pathophysiologic role for FFAs in inflammation and insulin resistance, it is intriguing that the lipid component of LPS is sufficient to trigger TLR4 signaling. In particular, a medium-chain fatty acid component of LPS, lauric acid, has been shown to initiate TLR4 signaling in a macrophage cell line (17-19). We therefore hypothesized that the obesity-induced increase in FFA levels can promote TLR4 signaling, which in turn contributes to obesitydependent insulin resistance. To test this hypothesis, we explored the role of TLR4 as a mediator of fatty acid actions on adipose cells/ tissue and macrophages. To assess the physiological role of TLR4 as a sensor of FFAs in vivo, we measured insulin signaling in muscle and insulin-sensitive glucose metabolism by hyperinsulinemic-euglycemic clamps following a 5-hour lipid infusion in TLR4-knockout and WT mice. We also investigated the role of TLR4 in energy balance and insulin resistance in response to chronic changes in dietary intake of fat by examining body weight, insulin sensitivity, and tissue expression of inflammatory mediators in TLR4-knockout mice on low-fat chow and high-fat diets.

\section{Results}

FFAs activate NF- $\mathrm{\kappa} B$ reporter in 293 cells and cytokine expression in macrophages via TLR4 signaling. Previous studies have demonstrated that lauric acid, a medium-chain fatty acid, can activate TLR4 signaling 


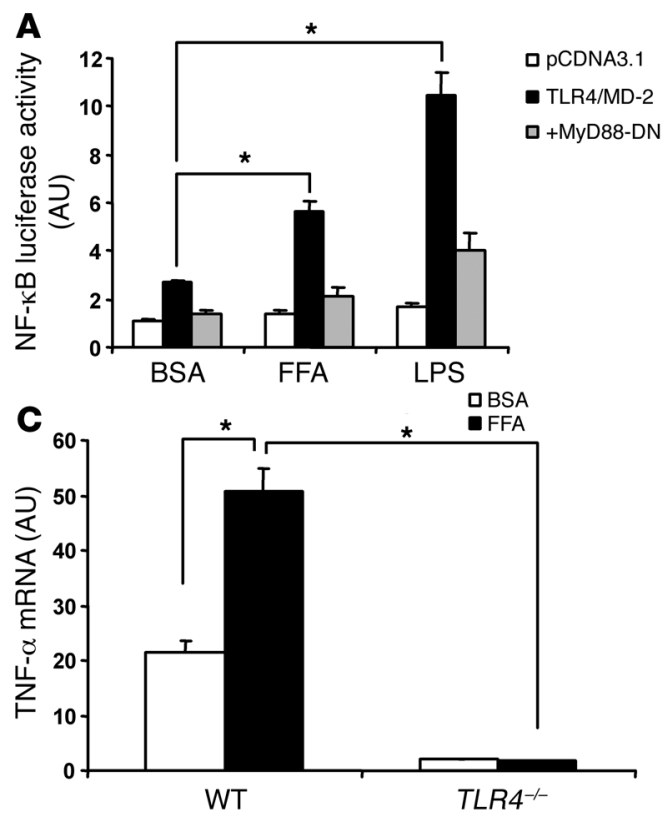

B
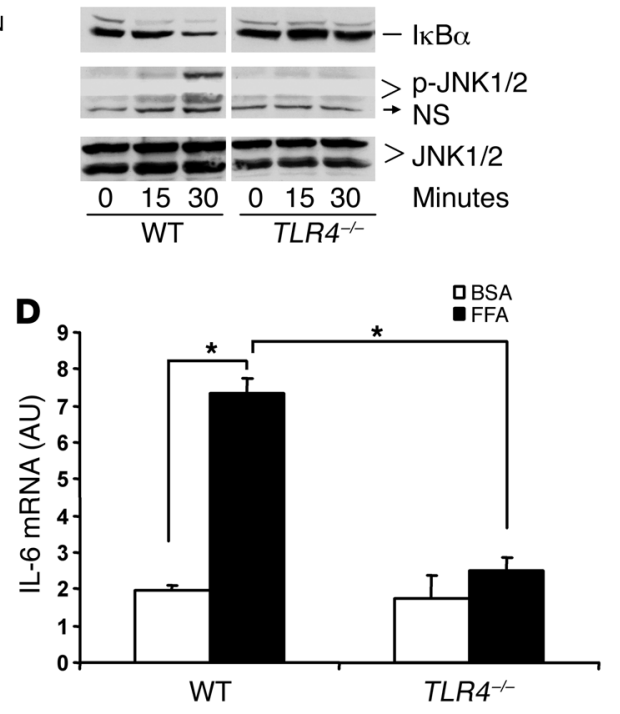

Figure 1

FFAs activate TLR4 signaling. (A) FFAs activate TLR4 signaling in transfected 293T cells $\left(n=6 ;{ }^{*} P<0.01\right)$. 293T cells were transiently transfected with TLR4/MD-2 expression vectors, with or without dominant negative MyD88 (MyD88-DN), and an NF-кB luciferase reporter and were then treated with a $200 \mu \mathrm{M}$ oleate/ palmitate mixture or $100 \mathrm{ng} / \mathrm{ml}$ LPS as a positive control. (B) FFAs cause $\mathrm{I} \kappa \mathrm{B} \alpha$ degradation and JNK phosphorylation in WT but not TLR4-deficient macrophages. Peritoneal macrophages were isolated and precultured for 4 days before treatment. Cells were treated with $500 \mu \mathrm{M}$ palmitate over the time course indicated. NS, nonspecific. (C and D) FFAs induce TNF- $\alpha$ and IL-6 mRNA in peritoneal macrophages in WT but not $T L R 4^{--}$mice $\left(n=4 ;{ }^{*} P<0.01\right)$. Peritoneal macrophages were treated with $200 \mu \mathrm{M}$ FFA mixture for 8 hours. Real-time RT-PCR was used to measure mRNA levels. Data are expressed as mean \pm SEM.

in a macrophage cell line $(17,18)$. We examined whether palmitate and oleate, 2 of the most abundant nutritional fatty acids, can also activate TLR4 signaling. 293T cells were transiently transfected with expression vectors for TLR4 and its cofactor MD-2, together with an NF-кB luciferase reporter construct, and were then treated with a $200 \mu \mathrm{M}$ oleate/palmitate mixture or $100 \mathrm{ng} / \mathrm{ml} \mathrm{LPS}$ as a positive control. Figure 1A shows that FFA treatment stimulated a doubling of NF- $\kappa \mathrm{B}$ luciferase activity compared with treatment with BSA control, mimicking LPS activation of NF- $\mathrm{KB}$ signaling. Most TLR4 signaling is mediated through MyD88, an immediate adaptor protein of TLR4 (15). Cotransfection of dominant-negative MyD88 substantially blocked both FFA and LPS activation of NF- $\mathrm{B}$ signaling (Figure 1A), indicating that FFA-induced TLR4 signaling is MyD88 dependent. To determine whether FFAs activate TLR4 signaling in macrophages with endogenous TLR4 expression, we isolated peritoneal macrophages from $T L R 4^{--}$and WT mice and treated these cells with FFAs. Figure 1B shows that FFAs caused the degradation of I $\mathrm{B} \alpha$ in WT macrophages but not in TLR4-deficient macrophages. Similarly, FFAs induced JNK phosphorylation in WT macrophages, while lack of TLR4 prevented this action (Figure 1B). Figure 1, C and D, shows that FFAs potently induced TNF- $\alpha$ and IL- 6 mRNA in WT macrophages but not in TLR4-deficient macrophages. Similar results were observed when cytokine protein release was measured in macrophages treated with FFAs (data not shown). Together, these data suggest that FFAs are capable of utilizing TLR4 signaling to induce an inflammatory response in macrophages.
To better assess the specificity of FFA action to induce inflammatory cytokine expression in macrophages, we treated cells of macrophage cell line RAW264.7 with various saturated, unsaturated, and trans fatty acids and examined cytokine expression. While trans fatty acids, arachidonic acid, and oleic acid induced slight increases in IL-6 mRNA expression, saturated fatty acids potently stimulated IL- 6 mRNA (Figure 2A). To further confirm the effects of saturated fatty acids on cytokine expression, we treated RAW264.7 cells with various doses of palmitic acid, using LPS treatment as a positive control. Figure 2B shows that palmitic acid stimulated TNF- $\alpha$ mRNA in a dose-dependent manner, mimicking LPS stimulation of TNF- $\alpha$ expression. Interestingly, omega-3 polyunsaturated fatty acids such as eicosapentaenoic acid (EPA) and docosahexaenoic acid (DHA) showed no effects on cytokine expression, and pretreatment of RAW264.7 cells with DHA completely inhibited saturated fatty acid-induced TNF- $\alpha$ mRNA expression (Figure 2C).

FFAs activate TLR4 signaling in adipose cells and tissue. We next investigated whether FFAs can utilize TLR4 to induce inflammatory signaling in adipocytes. It has been shown previously that adipocytes can exhibit features typical of macrophages, such as cytokine production and secretion $(1,2,7)$. Using Northern blots, we detected significant TLR4 mRNA expression in 3T3-L1 adipocytes, isolated mouse adipocytes, and adipose tissue, comparable to that in the unstimulated Raw 264.7 macrophage cell line (Figure $3 \mathrm{~A})$. To assess the potential relevance of TLR4 in mediating insulin resistance, we quantified TLR4 mRNA in adipose tissue of genetically obese/diabetic mice as well as diet-induced obese (DIO) mice. Figure 3, B and C, shows that TLR4 mRNA expression was increased in epididymal fat depots of $o b / o b, d b / d b$, and DIO mice. These data suggest that TLR4 mRNA expression is elevated in adipose tissue of obese/diabetic mice. Because TLR2, another member of the TLR family, has been reported to respond to certain fatty acids, we also evaluated TLR2 expression in 3T3-L1 adipocytes. Using real time RT-PCR, TLR2 mRNA was detectable in 3T3-L1 adipocytes and was markedly induced by treatment with LPS, a TLR4 agonist (Figure 3D). To test whether TLR4 and TLR2 are functional TLRs in adipocytes, we treated 3T3-L1 adipocytes with the TLR4 agonist LPS and the TLR2 agonist zymosan. While zymosan had no effect on IL-6 mRNA, LPS markedly stimulated IL-6 expression in 3T3-L1 adipocytes (Figure 3E). These data suggest that TLR4 expressed in adipocytes is functional in response to agonist treatment, whereas basal TLR2 expression is insufficient for cytokine induction. 

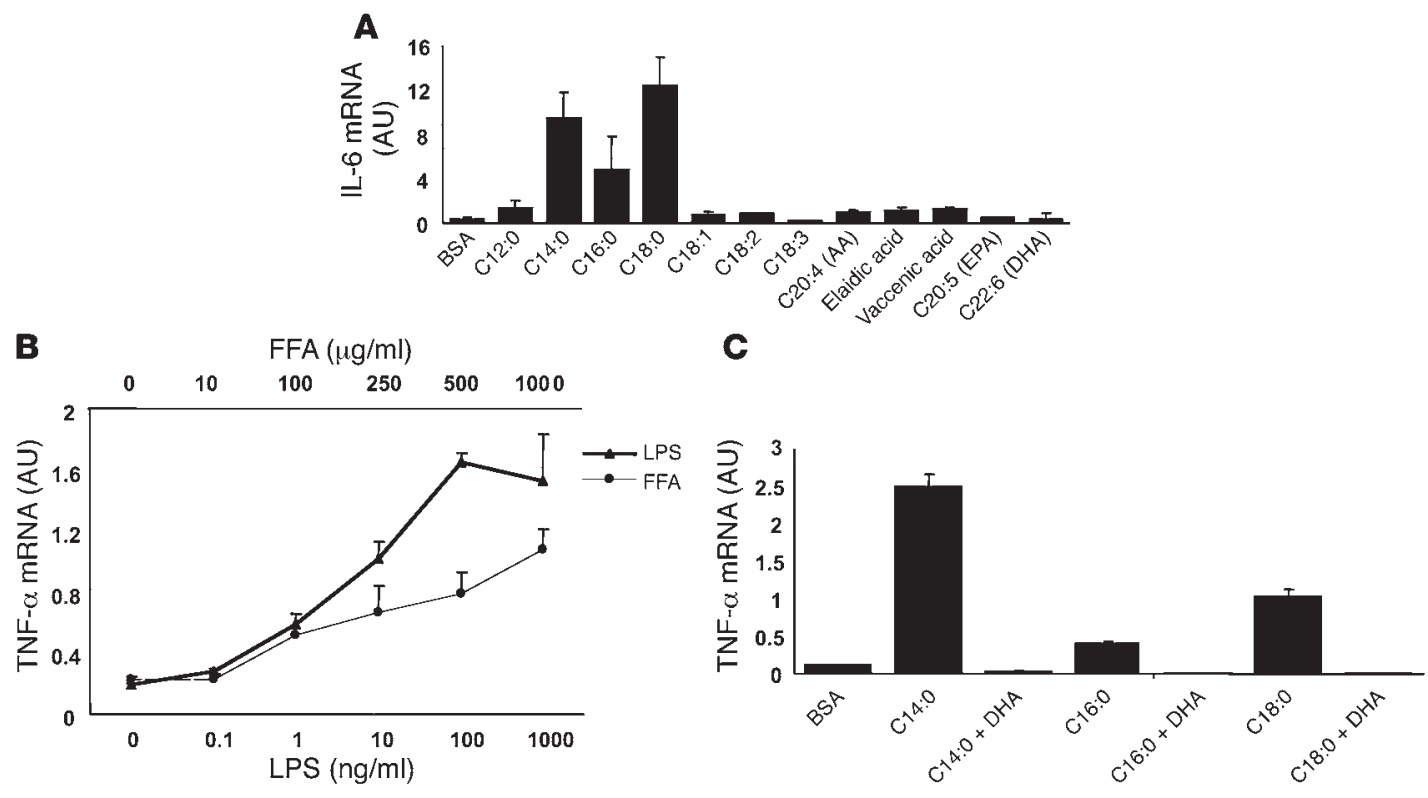

Figure 2

FFAs stimulate cytokine expression in macrophages. (A) Saturated FFAs induce IL-6 mRNA in the RAW264.7 macrophage cell line. Cells were treated with the indicated FFAs $(200 \mu \mathrm{M})$ for 8 hours. Saturated fatty acids include C12:0, C14:0, C16:0, and C18:0. AA, arachidonic acid; DHA, docosahexaenoic acid; EPA, eicosapentaenoic acid. (B) Palmitate stimulates TNF- $\alpha$ expression in a dose-dependent manner. RAW264.7 cells were treated with the indicated doses of palmitate or LPS for 8 hours. (C) The polyunsaturated fatty acid DHA blocks saturated FFA-induced TNF- $\alpha$ expression. RAW264.7 cells were pretreated with $200 \mu \mathrm{M}$ DHA for 2 hours and were then treated with $200 \mu \mathrm{M}$ of various saturated FFAs for 8 hours. Real-time RT-PCR was used to measure mRNA levels. Data are expressed as mean \pm SEM.

We then examined whether FFAs can induce inflammatory gene expression in adipocytes via TLR4. To this end, we reduced TLR4 expression in 3T3-L1 adipocytes by retroviral short hairpin RNA interference (shRNAi) knockdown. The TLR4 mRNA levels in the knockdown cells were suppressed by $78 \%$, as assessed by real-time RT-PCR (Figure 4A). We observed similar effects on TLR4 protein levels, measured by immunoblotting (Figure 4B). The TLR4 knockdown was specific, as TLR2 expression was not affected (Figure 4C). The knockdown adipocytes and control cells were then treated with $400 \mu \mathrm{M}$ of FFAs (palmitate and oleate mixture), with LPS used as a control. LPS potently stimulated IL-6 and TNF- $\alpha$ mRNA expression in 3T3-L1 adipocytes, while FFAs induced cytokine expression to a lesser extent (Figure 4, D and E). In contrast, TLR4 knockdown substantially prevented FFA/LPSinduced cytokine expression (Figure 4, D and E), indicating that FFAs cause an inflammatory response mediated by TLR4 in adipocytes. To study the role of endogenous TLR4 in mediating the FFA-induced inflammatory response in adipocytes, we isolated adipocytes from $\mathrm{TLR}^{-/-}$and WT mice and examined the cytokine expression and secretion in these cells in response to FFAs and LPS. Figure 4, F and G, shows that LPS significantly induces TNF- $\alpha$ and IL- 6 expression in WT adipocytes. FFAs were also capable of upregulating these 2 cytokines, although to a lesser extent than LPS. However, both FFA- and LPS-induced cytokine expression were substantially reduced in adipocytes from $T L R 4^{-/-}$mice. Similar results were observed with cytokine secretion (Figure 4, H and I). These data suggest that TLR4 participates in FFA-induced inflammatory response of adipocytes in culture.

To investigate the role of endogenous TLR4 in mediating FFAinduced inflammatory signaling in vivo, we infused $T L R 4^{--}$and WT mice with lipid (coupled with heparin to activate lipopro- tein lipase) to release FFAs into the circulation (Figure 5A) (13). We employed EMSA to examine the nuclear translocation and DNA binding of NF- $\kappa \mathrm{B}$, a downstream signaling target of TLR4 activation. An 8-hour lipid infusion markedly induced NF-кB DNA binding activity in nuclear extracts from adipose tissue of WT mice (Figure 5B, right panel), mimicking the NF- $\mathrm{B}$ DNA binding induced by LPS (Figure 5B, left panel). However, both lipid- and LPS-induced activation of NF- $\kappa B$ were prevented in adipose tissue of TLR4 $4^{-1-}$ mice (Figure $5 \mathrm{~B}$ ). To further confirm the lipid-induced activation of NF- $\kappa \mathrm{B}$, we combined chromatin immunoprecipitation (ChIP) assays with SYBR Green quantitative PCR analysis to examine in vivo NF- $\mathrm{B}$ binding to the consensus sequence of IL- 6 and monocyte chemoattractant protein- 1 (MCP-1) promoters in adipose tissue of mice infused with lipid or LPS. An 8-hour lipid infusion markedly increased NF-кB DNA binding to the consensus sequence within the IL- 6 promoter in adipose tissue of WT mice, mimicking LPS-induced NF-кB DNA binding to the IL- 6 promoter (Figure 5C). In contrast, TLR4 deficiency prevented both lipid- and LPS-induced NF-кB DNA binding to the IL- 6 promoter (Figure $5 \mathrm{C}$ ). Similar results were observed with NF-KB DNA binding to the MCP-1 promoter (data not shown). We further measured cytokine gene expression in adipose tissue of mice infused with lipid. Consistent with NF-кB activation, lipid infusion stimulated TNF- $\alpha$, IL- 6 , and MCP-1 mRNA expression in adipose tissue of WT mice. However, this effect was not seen in TLR $4^{-/-}$mice (Figure 5, D-F).

TLR4 deficiency ameliorates impaired insulin signaling in muscle and reduced insulin-mediated glucose metabolism induced by lipid infusion. To investigate the physiological consequence of lipid activation of TLR4 signaling, we examined insulin signaling in muscle and insulin sensitivity of glucose metabolism in vivo. After an 
A
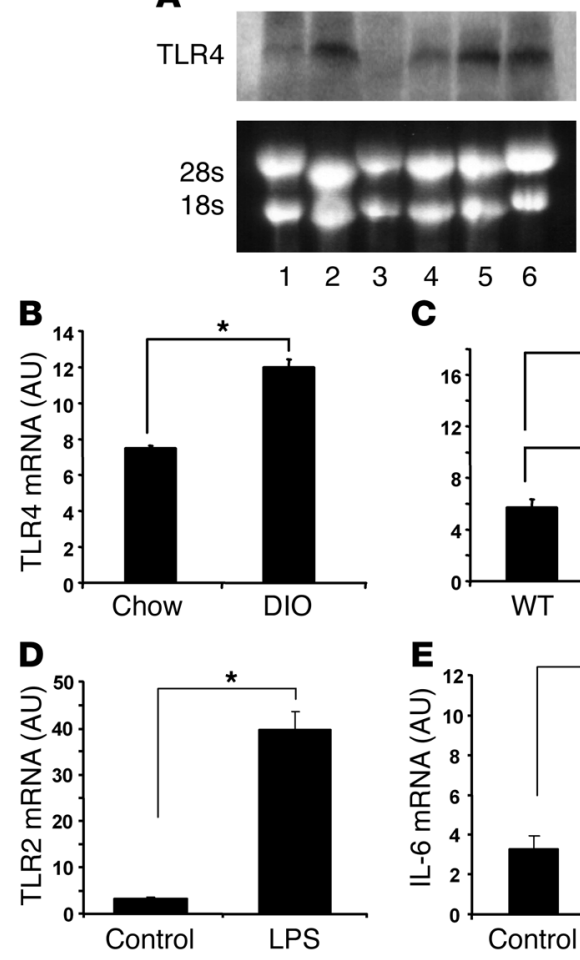

*
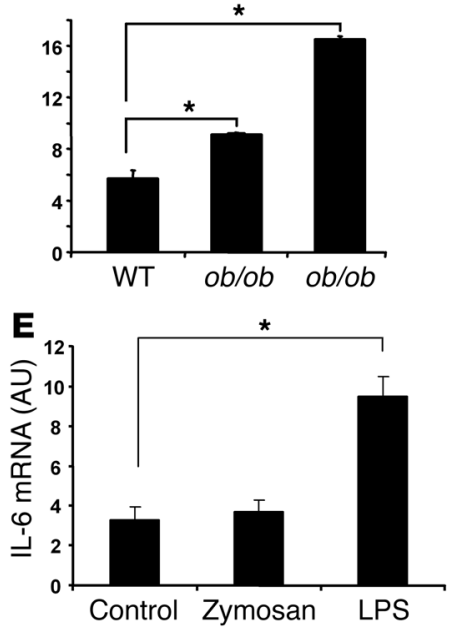

8-hour lipid infusion, mice were injected with insulin to acutely stimulate insulin signaling (Figure 6A). Lipid infusion markedly inhibited insulin-stimulated insulin receptor substrate-1 (IRS-1) phosphorylation in skeletal muscle of WT mice as indicated by Western blot analysis of immunoprecipitated muscle protein extracts using antibodies specific to tyrosine-phosphorylated IRS-1 protein (Figure 6B). This was substantially attenuated in TLR $4^{-/-}$mice. We further examined the docking of p85 to IRS-1 by immunoprecipitating IRS-1, followed by immunoblotting using anti-p85 antibodies. As with IRS-1 phosphorylation, lipid infusion markedly inhibited the docking of p85 to IRS-1 upon insulin injection in WT mice, while in $\mathrm{TLR}^{-{ }^{--}}$mice, p85 docking to IRS-1 was minimally affected by lipid infusion (Figure 6B). As serine kinases such as $\mathrm{iKK}$, normally activated in inflammatory signaling, may cause serine phosphorylation of IRS-1, resulting in the inhibition of IRS-1 tyrosine phosphorylation and impaired insulin signaling, we evaluated the serine phosphorylation of IRS-1 in skeletal muscle of mice infused with lipid. Figure 6C shows that lipid infusion markedly induced serine (Ser307) phosphorylation of IRS- 1 in WT mice, while this stimulation was greatly attenuated in $T L R 4^{-/-}$mice.

We further performed hyperinsulinemic-euglycemic clamp studies to examine the insulin sensitivity of glucose metabolism in $\mathrm{TLR}^{-/-}$and WT mice infused with either saline or lipid (Figure 7A). During a continuous insulin infusion at $2.5 \mathrm{mU} / \mathrm{kg} / \mathrm{min}$, the glucose infusion rate required to maintain euglycemia at $5.5 \mathrm{mM}$ was reduced by $65 \%$ in WT mice infused with lipid, compared with mice infused with saline, indicating that the lipid infusion induced insulin resistance. In contrast, lipid infusion exerted a lesser effect on the glucose infusion rate in TLR4 $4^{-/}$mice, suggesting that they were protected against insulin resistance (Figure 7B). Similarly, after lipid infusion, insulin-stimulated glucose

\section{Figure 3}

Adipocytes express functional TLR4, and TLR4 expression is increased in adipose tissue of obese models. (A) Adipocytes express TLR4. Northern blotting was used to detect TLR4 mRNA expression. Lane 1: 3T3-L1 preadipocytes; lane 2: 3T3-L1 adipocytes; lane 3: stromal-vascular cells; lane 4: mouse adipocytes; lane 5: mouse adipose tissue; lane 6: RAW264.7 macrophages. (B and C) TLR4 mRNA expression is increased in fat pads from DIO and $o b / o b$ and $d b / d b$ mice $\left(n=4 ;{ }^{*} P<0.05\right)$. TLR4 mRNA was measured using real-time RT-PCR. (D) The TLR4 agonist LPS induces TLR2 expression in adipocytes. (E) LPS but not zymosan stimulates IL-6 mRNA expression in 3T3-L1 adipocytes. 3T3-L1 adipocytes were treated with $100 \mathrm{ng} / \mathrm{ml}$ LPS or $40 \mu \mathrm{g} / \mathrm{ml}$ zymosan for 8 hours. Real-time RT-PCR was conducted to measure TLR2 and IL-6 mRNA levels. Data are expressed as mean $\pm \mathrm{SEM} ; n=6,{ }^{*} P<0.05$.

turnover was decreased by $40 \%$ in WT mice, while this reduction was substantially attenuated in $T L R 4^{-/-}$mice (Figure $7 \mathrm{C}$ ). Consistent with the measures of whole-body glucose turnover, lipid infusion decreased insulin-stimulated glucose uptake into skeletal muscle and fat of WT mice by $50 \%-60 \%$, while this effect was attenuated in TLR $4^{-/-}$mice (Figure $7, \mathrm{D}$ and E). Similarly, insulin-stimulated glycolysis appeared to be suppressed to a

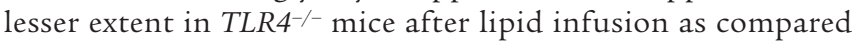
with WT mice (Figure 7F).

Effect of TLR4 expression on the response of mice to bigh-fat diets. Female TLR4 ${ }^{-/}$and WT mice were fed low-fat chow or high-fat diets over a period of 39 weeks. On chow diets, female TLR4 ${ }^{-/-}$ mice weighed significantly more, by an average of $3.6 \mathrm{~g}$ by 39 weeks, than WT mice (Figure 8A). On high-fat diets, TLR $4^{--}$mice showed a more rapid and greater weight gain (Figure 8A). Elevated body weights were observed by 3 weeks after the start of the diet, and the mean difference between genotypes was $9.2 \mathrm{~g}$ by 39 weeks. As assessed by dual energy x-ray absorptiometry, this increased weight was attributable to increased fat mass, with no change in lean mass (Figure 8B), and was caused by increased food intake (Figure 8C), as there was no detectable change in energy expenditure as assessed by metabolic chamber analysis

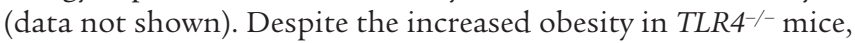
insulin tolerance tests suggested that these mice were somewhat more insulin sensitive, rather than more insulin resistant, than WT mice (Figure 8D). To explore the molecular basis for this protection from insulin resistance, we examined the expression of inflammatory genes in response to high-fat diets. While in WT mice, high-fat diet-induced adipose tissue expression of TNF- $\alpha$, IL-6, SOCS3, MCP-1, and the macrophage marker F4/80, this induction was greatly attenuated in $T L R 4^{-/-}$mice (Figure 8E). Similar findings were obtained for gene expression in liver (Figure $8 \mathrm{~F}$ ). In male mice on chow or high-fat diets, there were no differences between genotypes in body weight, food intake, or insulin sensitivity (data not shown). Nonetheless, analysis of gene expression revealed blunting of high-fat diet-induced inflammatory gene expression in adipose tissue and liver of male TLR4 $4^{-/-}$mice (data not shown).

\section{Discussion}

This article addresses the hypothesis that TLR4, the obligatory receptor for bacterial LPS and a key molecular component of the innate immune system, can play an additional role as well, as a "sensor" for endogenous lipids that may contribute to the pathogenesis of lipid-induced insulin resistance. The plausibility of this 

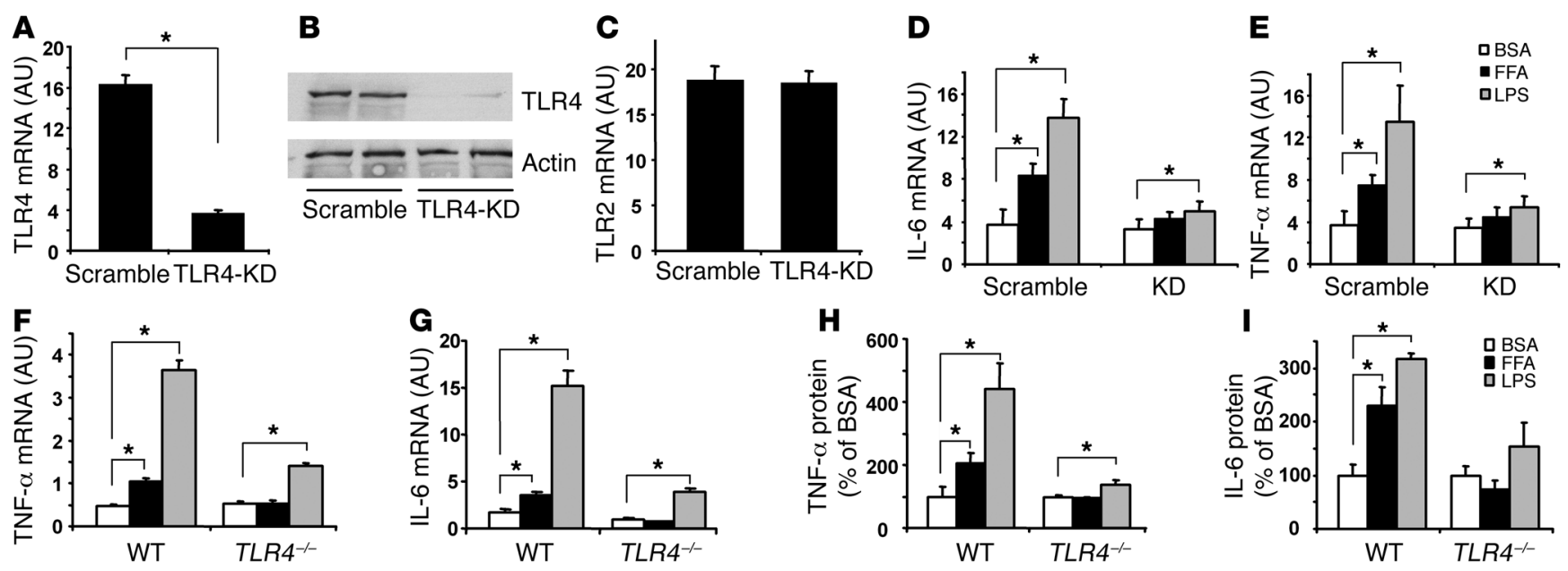

Figure 4

FFAs cause inflammatory response via TLR4 in adipocytes. (A-C) Generation of an adipocyte model with specific TLR4 knockdown. 3T3-L1 preadipocytes were infected with retroviral short hairpin RNA interference (shRNAi) to knock down TLR4 (TLR4-KD), and cells were selected and then differentiated into adipocytes. TLR4 mRNA (A) and protein (B) and TLR2 mRNA (C) levels were evaluated by real-time RT-PCR and immunoblotting. Data are expressed as mean \pm SEM; $n=6$; ${ }^{*} P<0.05$. (D and E) FFAs stimulate IL-6 and TNF- $\alpha$ mRNA expression in 3T3-L1 adipocytes via TLR4. TLR4-knockdown and scramble control adipocytes were treated with $400 \mu \mathrm{M}$ FFA (palmitate and oleate mixture) or $100 \mathrm{ng} / \mathrm{ml}$ LPS for 12 hours. Real-time RT-PCR was conducted to measure the mRNA levels. $n=4 ;{ }^{*} P<0.05$. (F and G) FFAs stimulate TNF- $\alpha$ and IL-6 mRNA in WT but not in TLR4 ${ }^{-/}$adipocytes $\left(n=4\right.$; $\left.{ }^{*} P<0.05\right)$. (H and I) FFAs stimulate TNF- $\alpha$ and IL-6 protein secretion in WT but not in TLR4 ${ }^{-/-}$ adipocytes $\left(n=4 ;{ }^{*} P<0.05\right)$. Mouse adipocytes were isolated and precultured for 6 hours and then were treated with $400 \mu \mathrm{M}$ FFA mixture for 16 hours. Real-time RT-PCR was used to measure mRNA levels. Data are expressed as mean \pm SEM.

hypothesis derives from several prior observations. First, obesity and diabetes have been shown, through a variety of approaches, to be associated with activation of inflammatory pathways in key metabolic tissues as well as macrophages $(1,2,7,12,14,20-23)$, and these inflammatory signals and their downstream effectors have been implicated as mediators of resistance to insulin action that characterize these states $(1,2,7)$. FFAs, whose levels are commonly increased in obesity, have been implicated as proximate causes of insulin resistance in several models $(8-14,24,25)$ and have also been shown to be capable of inducing inflammatory signaling in several tissues (9, 26-32). FFAs have been shown to cause insulin resistance in vivo - as evidenced by their impairment of insulin's ability to activate signal transduction, inhibit hepatic glucose production, and stimulate glucose uptake in skeletal muscle and adipose tissue $(8,9,13,25-27,33)$. The molecular pathways by which FFAs induce insulin resistance have been investigated in insulin-sensitive tissues such as fat and skeletal muscle. FFAs have been shown to activate JNK and stimulate TNF- $\alpha$ expression in 3T3-L1 adipocytes, while blockage of JNK or TNF- $\alpha$ can prevent FFA-induced insulin resistance, suggesting that fatty acid-induced insulin resistance may be mediated in part by these proinflammatory signaling pathways (26). Moreover, FFAs also activate the proinflammatory signal IKK/ NF- $\mathrm{B}$ in 3T3-L1 adipocytes, leading to expression of the cytokines TNF- $\alpha$ and IL- $6(27,28)$. However, the critical question of how FFAs are sensed by adipocytes or other tissues in order to activate proinflammatory signaling networks remains uncertain. Whether such sensing involves an intracellular target of FFA, is a consequence of FFA metabolism to a subsequent mediator, or requires an action of FFA at the plasma membrane has not been determined.

Several plausible theories have been advanced to explain the ability of FFAs to activate intracellular inflammatory signals. These include uptake and intracellular actions of FFAs, either through their subsequent metabolism to species such as long-chain CoA derivatives or via their action as ligands to modify nuclear receptors. Proof that such mechanisms underlie fatty acid-induced inflammatory signaling has yet to be provided. An additional or alternative mechanism might involve actions of FFAs to modify plasma membrane pathways that are capable of initiating inflammatory signals. Several G protein-coupled receptors have recently been shown to respond to FFAs as a ligand $(34,35)$, but these have not been implicated in FFA-induced inflammation or insulin resistance. TLRs, including TLR4, are potential candidates for such a role, as these receptors are known to initiate signals that activate NF-KB, JNK, and SOCS pathways $(15,16,19)$, all of which participate in regulation of inflammation and have been proven as well to be capable of inducing insulin resistance $(12,14,20$, $21,36)$. In this regard, it has been shown that the C12 fatty acid lauric acid, a major component of lipid A of LPS, is capable of activating TLR4 signaling $(17,18)$. Nutritional fatty acids have been shown, using in vitro systems, to activate TLR4 signaling as well $(17,18)$. Thus, multiple lines of evidence converge on the possibility that FFAs might initiate inflammatory signaling and insulin resistance, at least in part, by activating TLR4 signaling in one or more tissues and that such signaling might contribute to insulin resistance, or at least that component related to FFAs. In this study, we have combined a number of in vitro and in vivo approaches to assess this hypothesis.

The function of TLR4 has been most extensively studied in cells of the immune system, including macrophages. The relevance of macrophages to the pathogenesis of type 2 diabetes was not previously viewed as likely. However, over the past several years, a potential role for macrophages as mediators of insulin resistance has emerged from elegant genetic studies (21-23), so potential activation of macrophage TLR4 by FFAs can now be readily linked to 


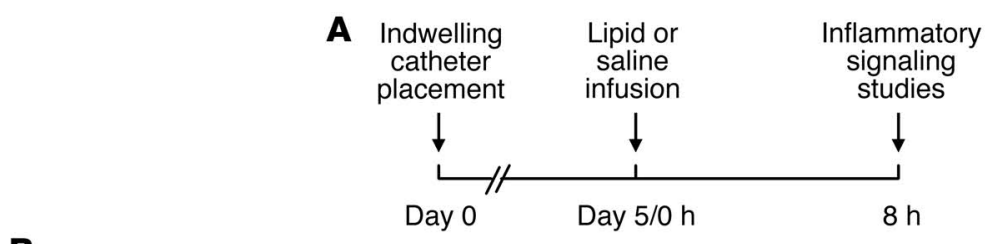

B
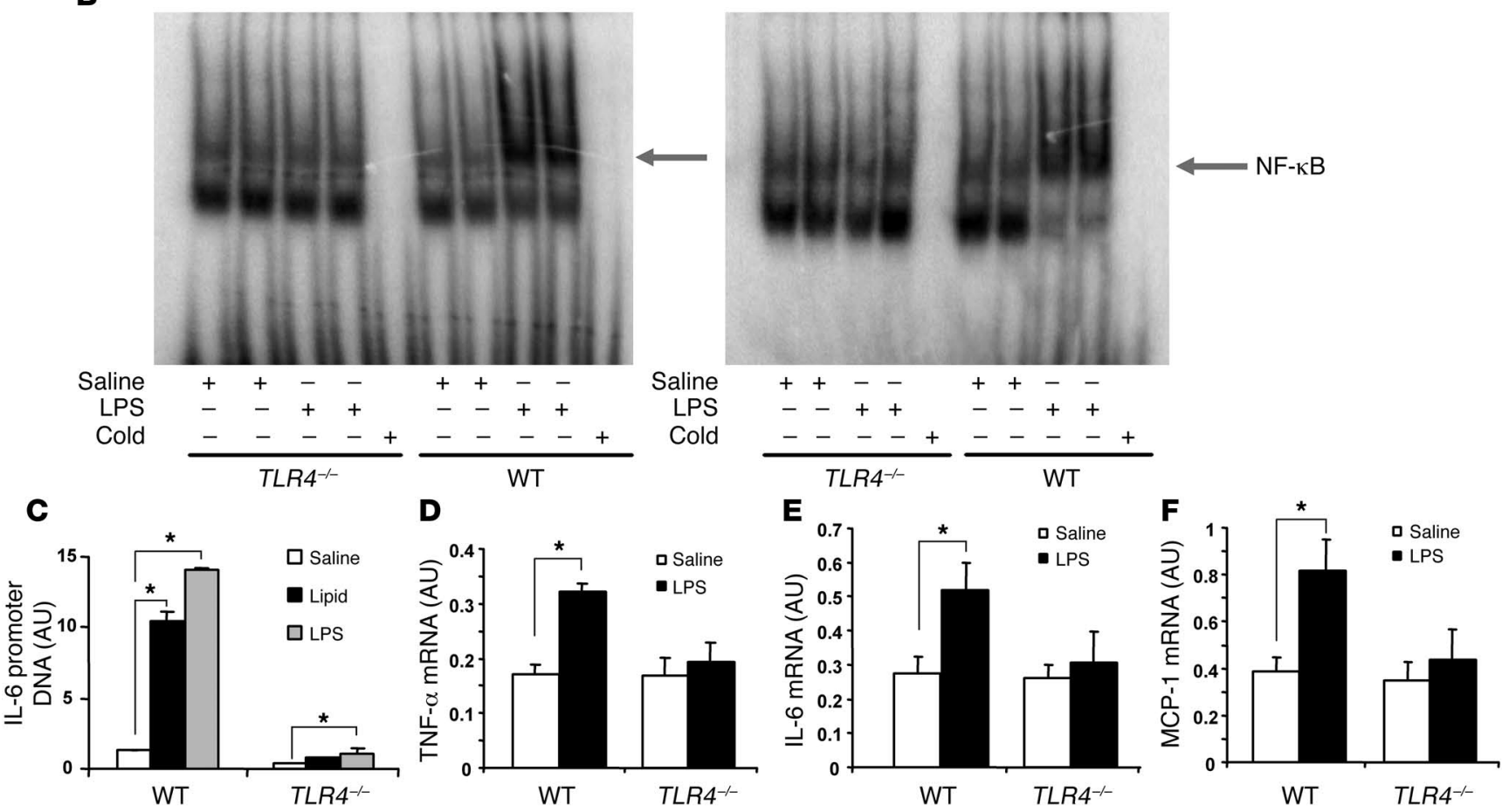

Figure 5

Lipid infusion activates TLR4 signaling in adipose tissue in vivo. (A) Schematic diagram of lipid infusion and inflammatory signaling studies. (B) Lipid infusion increases NF-кB DNA binding in adipose tissue of WT but not TLR4-- mice. Mice were fasted overnight and infused with lipid (coupled with heparin) for 8 hours. EMSA was conducted to examine the NF-кB DNA binding activity. (C) Lipid infusion increases NF-kB DNA binding to the IL-6 promoter. ChIP assays were conducted to quantify NF-KB DNA binding to the IL-6 promoter. SYBR Green quantitative PCR was used to measure the immunoprecipitated DNA $\left(n=3 ;{ }^{*} P<0.01\right)$. (D-F) Lipid infusion increases TNF- $\alpha$, IL-6, and MCP-1 mRNA expression in adipose tissue of WT but not $T L R 4^{-/-}$mice $\left(n=4 ;{ }^{*} P<0.05\right)$. Data are expressed as mean \pm SEM.

insulin resistance. In addition, to the extent that TLR4 is expressed in tissues directly involved in insulin's metabolic actions, such as adipose tissue and muscle, an ability of FFAs to activate TLR4 in these tissues could be important as well. Our findings in this study are relevant to both of these possibilities.

We have demonstrated that a mixture of palmitate and oleic acid acts through TLR4 to induce NF- $\mathrm{BB}$ signaling in 293 cells, confirming earlier observations $(17,18)$. In addition, we have shown that palmitate acts through TLR4 on mouse peritoneal macrophages to induce IкB $\alpha$ degradation and activate JNK. We have also addressed the possibility that TLR4 is expressed on adipocytes and can mediate inflammatory signaling in this cell type. Our findings indicate that TLR4 is expressed on the 3T3-L1 adipocytes in a differentiation-dependent manner and is expressed in normal adipose tissue. The level of expression is similar to that of unstimulated RAW cells. The expression of TLR 4 mRNA in total adipose tissue extracts increased in 2 models of obesity, and this could be due in part to increased numbers of macrophages known to reside in fat tissue of obese animals. In 3T3-L1 adipocytes, FFAs induced increased expression of SOCS3 and IL-6
mRNA. In isolated mouse adipocytes, FFAs induced release of TNF- $\alpha$ and IL-6, and, importantly, this action of FFA was not observed in adipocytes from TLR $4^{-/}$mice.

To further examine the role of TLR4 in lipid-mediated inflammatory signaling in an in vivo setting, we utilized the model of i.v. infusion of lipid plus heparin. This model has been used repeatedly to demonstrate the ability of acute elevation of lipids in vivo to suppress insulin-stimulated glucose metabolism and insulin signaling $(10,11,13)$. In this system, a 5-hour lipid infusion in mice reduces the action of insulin to stimulate glucose disposal into muscle and fat and suppresses insulin-mediated signal transduction $(10,11,13)$. In agreement with previous results, lipid infusion suppressed insulin-mediated glucose disposal, as measured by euglycemic clamp, and glucose uptake into muscle and fat, as assessed by 2-deoxyglucose uptake in WT mice. In contrast, in mice lacking TLR4, this adverse effect of lipid infusion was markedly suppressed.

To study the mechanism for the observed reduction in lipidinduced insulin resistance in TLR4 $4^{-/}$mice, we assessed the ability of lipid infusion to induce inflammatory signaling in adipose tissue. 
A

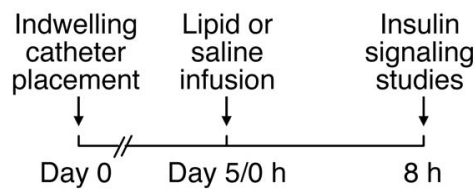

B

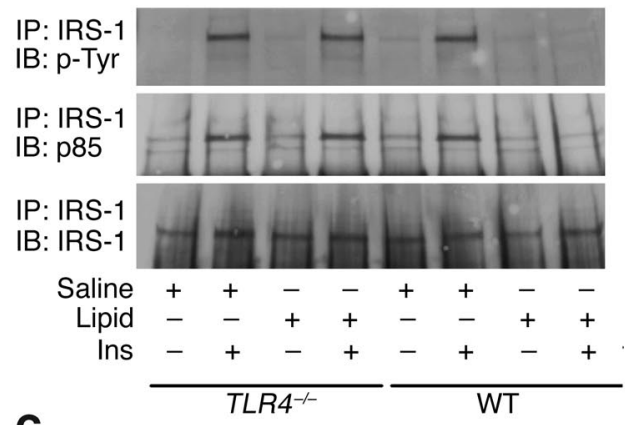

C

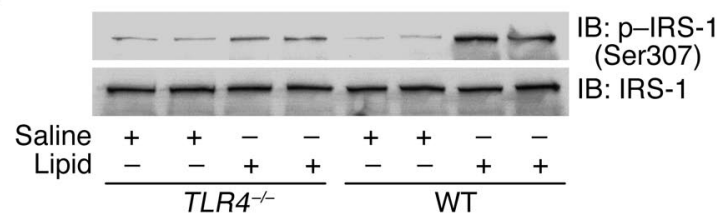

We found 2 indications that lipid infusion activated NF-кB signaling in adipose tissue, and this did not occur in TLR4-/mice. First, we determined by EMSA analysis that lipid infusion activated NF-KB in adipose tissue. Second, we used ChIP assays to show that lipid infusion induced binding of NF- $\kappa \mathrm{B}$ to sequences in the promoter of IL- 6 and MCP- 1 within adipose tissue. Strikingly, this did not occur in mice lacking TLR4 in both cases. Given the time course, it is most likely that this was due to activation of NF-KB in adipocytes, but these findings are also consistent with activation of macrophages either resident in adipose tissue or recruited to adipose tissue during the course of the infusion. The potential biologic significance of the findings for insulin resistance in adipose tissue could be similar in either case. The finding that lipid infusion reduced the ability of insulin to acutely activate signaling through IRS-1 likely underlies the reduced glucose uptake into muscle after lipid infusion. As TLR4 expression on muscle cells has been described $(37,38)$, this is likely a direct effect of lipid infusion on muscle cells, though indirect effects may also be present.

Having demonstrated both in vitro and in vivo that FFAs can act through TLR4 on macrophages and adipocytes to induce inflammatory signaling and suppress insulin signaling and insulin-mediated regulation of glucose metabolism, we next sought to determine the impact of TLR4 on high-fat diet-induced obesity and metabolic sequelae. In female mice, TLR4 status had a clear effect on energy homeostasis, with increased weight gain and fat mass, exaggerated by high-fat diet. This was attributed to increased food intake rather than changes in energy expenditure. Despite the increased obesity linked to TLR4 deficiency, insulin tolerance tests in female TLR4 ${ }^{-/}$mice showed improved insulin sensitivity. Consistent with the initial hypothesis of our studies, this protection from high-fat diet-induced insulin resistance was associated with failure of high-fat diet to induce expression of inflammatory mediators or indicators in liver and fat. These data suggest that, in female $\mathrm{C} 57 \mathrm{BL} / 6 \mathrm{~J}$ mice, TLR4 is in part required for the ability

\section{Figure 6}

TLR4 deficiency prevents impaired insulin signaling in skeletal muscle caused by lipid infusion. (A) Schematic diagram of lipid infusion and in vivo insulin signaling studies. (B) Mice preinfused with lipid for 8 hours were i.v. injected with $10 \mathrm{U} / \mathrm{kg} \mathrm{BW}$ of human insulin (Ins) or saline. Muscle lysates were immunoprecipitated and then immunoblotted with antibodies as indicated. (C) Mice were infused with lipid for 8 hours. Muscle lysates were immunoblotted with antibodies as indicated. Ser, serine; Tyr, tyrosine.

of high-fat diet to induce inflammatory mediators in peripheral tissues and may thereby contribute as well to the insulin resistance that develops. The results do not support the conclusion that TLR4 is the exclusive mechanism, however, as insulin resistance of lesser degree was seen after high-fat diet in $T L R 4^{-/}$mice. The fact that these changes in energy balance and insulin sensitivity were not evident in male mice despite suppression of inflammatory markers indicates that TLR4 and these inflammatory mediators are among a larger set of factors involved in linking diet, obesity, and insulin resistance, and these factors are subject to further modification by sex-specific factors. In addition, our data strongly suggest that while lipid infusion protocols can provide valuable insights into potential mechanisms for lipid-induced changes in insulin signaling and insulin resistance, this model is not necessarily predictive of the findings in mice fed high-fat diets. Whether the differences between these models are the result of different circulating FFA levels, the nature of specific fatty acids involved, variable duration of exposure, or other factors remains to be determined. With respect to FFA levels, our data closely resemble those of Kim et al. (39), who found that mean FFA levels were elevated 4-fold by high-fat diet but 5- to 6-fold after lipid infusion.

Additional study is needed to determine the cells/tissues in which TLR4 signaling is required for regulation of systemic insulin sensitivity. FFAs cause inflammatory responses in insulin-sensitive tissues such as skeletal muscle, resulting in both local and systemic insulin resistance. Two inflammatory kinases, PKC- $\theta$ and IKK $\alpha$, appear to play roles in fatty acid-induced insulin resistance in muscle, since PKC- $\theta$ or IKK $\alpha$ knockout or inactivation of IKK $\alpha$ by high-dose salicylate can prevent FFAinduced defects in insulin signaling and insulin resistance in skeletal muscle $(10,11)$. In a human study, lipid-induced insulin resistance was associated with activation of IKK/NF- $\mathrm{KB}$ signaling in skeletal muscle (29). Further studies confirmed that FFAs activate IKK/NF- $\mathrm{KB}$ signaling in myotubes, leading to cytokine expression $(30,31)$. Since skeletal muscle cells express functional TLR4 that activates proinflammatory signaling in response to LPS $(37,38)$, it is possible that FFA-induced insulin resistance in skeletal muscle is mediated by FFA activation of TLR4 expressed on muscle cells. Indeed, our data show that TLR4 deficiency substantially limits impaired insulin signaling and insulin resistance caused by lipid infusion in muscle. However, additional studies, involving, for example, specific deletion of TLR4 in individual tissues, will be required to determine to what extent the improved insulin sensitivity in response to FFAs in a given tissue such as muscle is due to the direct effect of TLR4 deficiency in that tissue, as opposed to systemic effects secondary to changes exerted in adipose tissue or macrophages.

The obesity observed in female $T L R 4^{-/-}$mice is worthy of discussion. The mechanism involves changes in food intake and presumably indicates some changes in the CNS circuitry involved in 
A
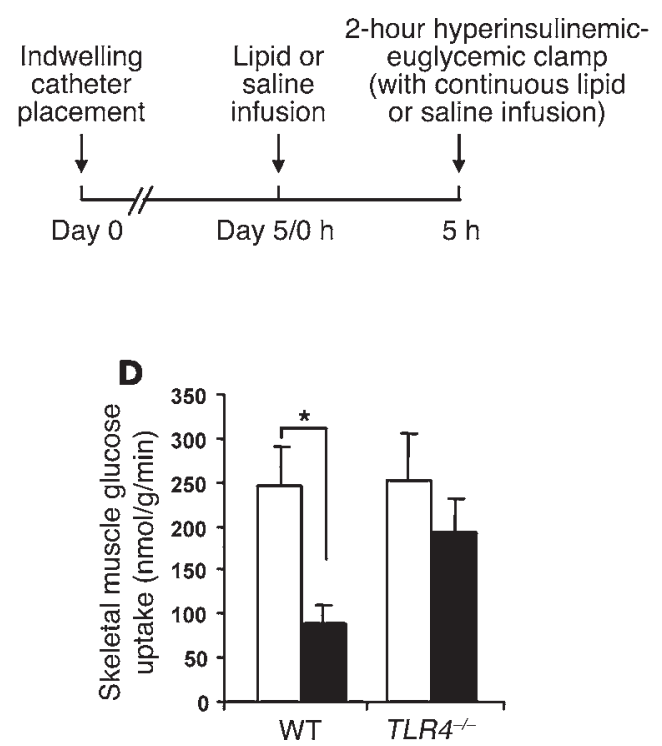

B
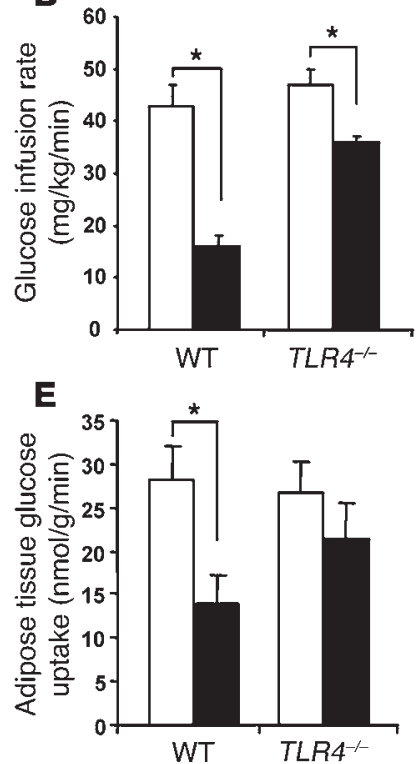

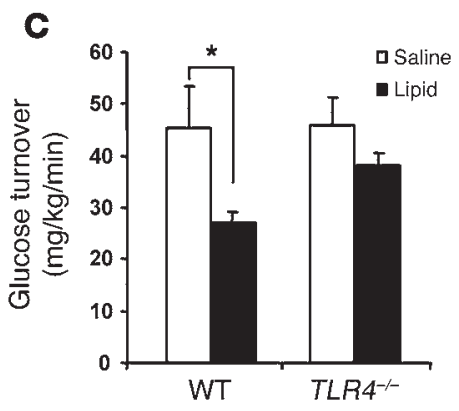

$\mathbf{F}$

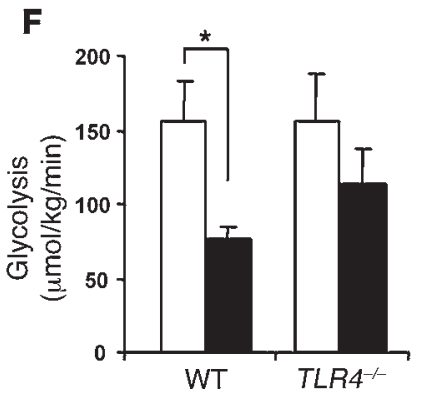

Figure 7

TLR4 deficiency prevents lipid-induced insulin resistance. Mice were fasted overnight and preinfused with lipid for 5 hours. (A) Schematic diagram of lipid infusion and hyperinsulinemic-euglycemic clamp studies. Hyperinsulinemic-euglycemic clamps were conducted to examine the

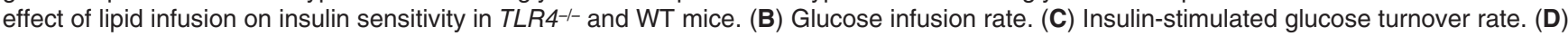
Insulin-stimulated glucose uptake in skeletal muscle (gastrocnemius). (E) Insulin-stimulated glucose uptake in white adipose tissue (epididymal fat). (F) Insulin-stimulated whole-body glycolysis. All data are expressed as mean \pm SEM; $n=4-10 ;{ }^{*} P<0.05$.

appetite regulation. At this time, it is unclear by what mechanism this arises secondary to TLR4 deficiency. It is possible that TLR4 deficiency in a peripheral tissue results in altered levels of a systemic regulator of appetite or that TLR4 deficiency in CNS has a more direct role. TLR4 expression has been identified in several brain regions (40), but whether such receptors play a role energy balance will require additional studies.

In summary, although FFAs are known to play a key role in the etiology of insulin resistance, the molecular sensing mechanisms through which high levels of FFAs are transduced to produce intracellular inflammatory signaling, which then induces insulin resistance, have remained obscure. We have shown here that FFAs are capable of utilizing the innate immune receptor TLR4 to induce proinflammatory cytokine expression in macrophages, adipocytes, and liver. Moreover, TLR4 signaling appears to be required for a component of insulin resistance induced by FFAs in adipocytes and in vivo after lipid infusion and high-fat diets. These data therefore suggest a previously unappreciated link between the innate immune system and metabolism and suggest that TLR4 may be involved in other adverse effects of FFAs on tissues and processes that constitute the metabolic syndrome.

\section{Methods}

Animals. TLR4-knockout (TLR4-/) mice (41) were kindly provided by D.T. Golenbock (University of Massachusetts, Worcester, Massachusetts, USA) and were backcrossed 6 generations into the C57BL6/J strain. WT littermates were used as controls. The 16 -week-old $o b / o b$ and $d b / d b$ mice were purchased from the Jackson Laboratory. For DIO mice, C57BL/6J mice were fed high-fat diets (D12331; Research Diets Inc.) for 16 weeks. The animal studies were approved by the Institutional Animal Care and Use Committee of Beth Israel Deaconess Medical Center.
Cell culture. Murine 3T3-L1 preadipocyte culture and differentiation were conducted as previously described (36). For the TLR4 siRNA knockdown study, 3T3-L1 preadipocytes were infected with retroviral siRNA, which was constructed according to the manufacturer's manual (RNAi-Ready pSIREN-RetroQ Vector; BD Biosciences - Clontech). Cells were selected with puromycin and then differentiated into adipocytes. Primary-cultured adipocytes were also isolated and cultured as previously described (41), with the isolated adipocytes being precultured for 6 hours before treat-

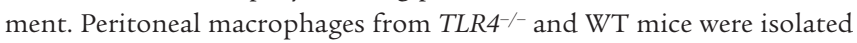
by lavage 4 days after i.p. injection of $3 \%$ thioglycollate $(2.5 \mathrm{ml}$; Difco; BD Diagnostics) (42). The cells were plated at a density of $1.2 \times 10^{6}$ cells/well in 6-well plates and cultured in RPMI medium containing $10 \%$ FBS. Fatty acids (oleate and palmitate mixture; Sigma-Aldrich) were conjugated with BSA at a 4:1 molar ratio before treatment. Palmitate was dissolved in $95 \%$ ethanol at $60^{\circ} \mathrm{C}$ and then was mixed with prewarmed BSA (10\%) to yield a stock concentration of $7.5 \mathrm{mM}$. The endotoxin content of BSA-fatty acid conjugates was measured with the Chromogenic Limulus Amebocyte Lysate (LAL) Test (Cambrex). The BSA-fatty acid preparations contained less than $0.04 \mathrm{ng} / \mathrm{ml}$ endotoxin, which in our studies did not cause significant inflammatory signaling in either macrophages or adipocytes. LPS from Escherichia coli 0127:B8 was purchased from Sigma-Aldrich.

The IL- 6 and TNF- $\alpha$ protein secreted into medium were measured using mouse IL- 6 and TNF- $\alpha$ ELISA kits (R\&D Systems).

Transfection and luciferase reporter assay. Murine TLR4, MD-2, and MyD88 cDNA constructs were kindly provided by R. Medzhitov (Yale University, New Haven, Connecticut, USA), D.T. Golenbock (University of Massachusetts, Worcester, Massachusetts, USA), and J.T. Schopp (University of Lausanne, Epalinges, Switzerland), respectively. These cDNA constructs were further subcloned into pcDNA3.1 expression vectors (Invitrogen). pNFKB-Luc vectors were purchased from BD Biosciences - Clontech. $293 \mathrm{~T}$ cells were transfected with expression vectors using a SuperFect 

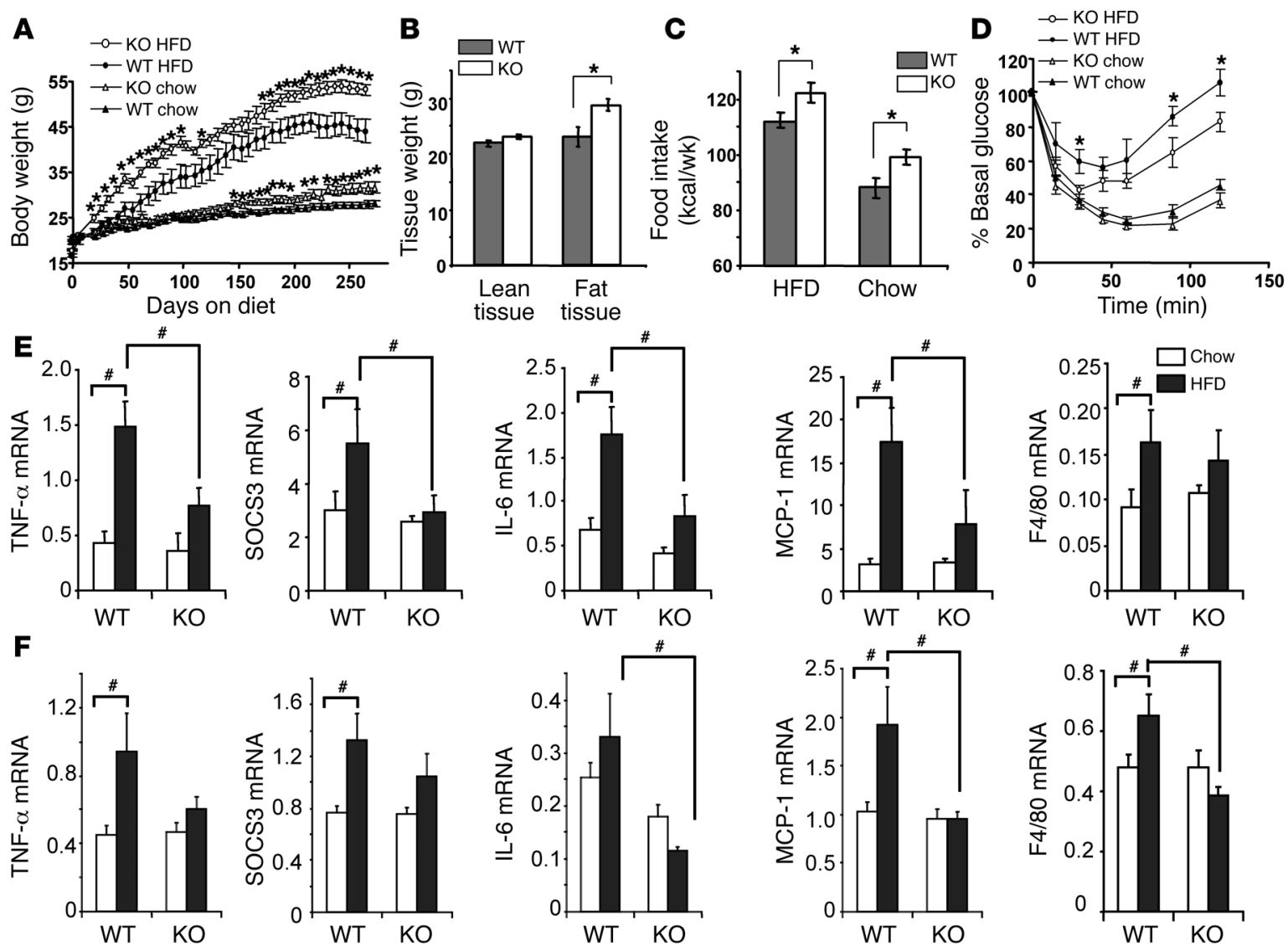

Figure 8

Female mice lacking TLR4 show increased obesity but are partially protected against high-fat diet-induced (HFD-induced) insulin resistance, and HFD does not induce inflammatory gene expression in fat and liver in these mice. (A) Body weights of WT and TLR4 ${ }^{--}$mice on HFD or chow diet. (B) Lean and fat tissue weight as assessed by dual-energy x-ray absorptiometry at 26 weeks on HFD. (C) Cumulative food intake. Daily food intake was measured for 1 week after 22 weeks on diet. (D) Insulin tolerance test. Insulin (1 mU/g BW) was administered to mice after 36 weeks on diet. HFD induces inflammatory gene expression in fat $(\mathbf{E})$ and liver $(\mathbf{F})$ in WT but not TLR4-knockout mice. Data are expressed as mean \pm SEM $(n=7-9)$. ${ }^{*} P<0.05$, WT versus TLR4 ${ }^{--}$; ${ }^{\#} P<0.05$ between groups as indicated.

Transfection Reagent kit (QIAGEN), and luciferase activity was measured using a Dual-Luciferase Reporter Assay kit (Promega), according to the manufacturer's instructions.

Total RNA extraction, quantitative RT-PCR, and Northern blots. Adipose tissue, skeletal muscle, and macrophage total RNA was extracted using an RNeasy Mini Kit (QIAGEN) or Tri Reagent kit (Molecular Research Center) and DNase I treatment (QIAGEN), according to the manufacturer's protocol. mRNA expression of inflammatory genes was assessed by quantitative RT-PCR (Brilliant Single-Step, Stratagene) using a Stratagene Mx 4000 thermocycler, as previously described (36). The DNA sequence of primers and probes corresponding to mouse inflammatory genes is as follows: TNF- $\alpha$, forward, GACCCTCACACTCAGATCATCTTCT, reverse, CCACTTGGTGGTTTGCTACGA, probe, AAAATTCGAGTGACAAGCCTGTAGCCCA; MCP-1, forward, GGCTCAGCCAGATGCAGTTAA, reverse, CCTACTCATTGGGATCATCTTGCT, probe, CCCCACTCACCTGCTGCTACTCATTCAC; TLR4, forward, GCAGAAAATGCCAGGATGATG, reverse, AACTACCTCTATGCAGGGATTCAAG, probe, CCTGGCTAGGACTCTGATCATGGCACTGT. IL- 6 primer and probe pair was purchased from Applied Biosystems. Northern blots were conducted as previously described (43).

Hyperinsulinemic-englycemic clamps. Hyperinsulinemic-euglycemic clamp experiments were conducted as previously described (13). The procedure is summarized in Figure 1. Three-month-old male TLR4-- and littermate WT mice with an average body weight of $30 \mathrm{~g}$ were implanted with indwelling catheters (Micro-Renathane tubing; MRE-025; Braintree Scientific Inc.) in the right jugular veins. After a 5-day recovery, overnight-fasted mice were preinfused with lipid (5 ml/kg/h; Liposyn II; Abbott) and heparin $(6 \mathrm{U} / \mathrm{h})$ or saline for 5 hours with a microdialysis pump (CMA/102; CMA Microdialysis). After an initial $5-\mu \mathrm{Ci}$ bolus, $\left[3{ }^{3} \mathrm{H}\right]$ glucose (PerkinElmer) was infused at $0.05 \mu \mathrm{Ci} / \mathrm{min}$ for 2 hours to measure basal glucose turnover. With the continuous infusion of lipid or saline, a 2-hour hyperinsulinemic-euglycemic clamp was conducted with a primed and continuous infusion of human insulin (Humulin; Lilly) at a rate of $2.5 \mathrm{mU} / \mathrm{kg} / \mathrm{min}$, coupled with a variable infusion of $20 \%$ glucose to maintain blood glucose concentrations at $5.5 \mathrm{mM}$. The rate of whole-body glucose turnover was estimated using a continuous infusion of $\left[3-{ }^{3} \mathrm{H}\right]$-glucose at $0.1 \mu \mathrm{Ci} / \mathrm{min}$ after a $10-\mu \mathrm{Ci}$ bolus. Blood was collected during the hyperinsulinemic period for measurement of whole-body glucose turnover and glycolysis, which were determined as previously described (13). Tissue-specific glucose uptake was estimated by a bolus administration of $10 \mu \mathrm{Ci} 2$-deoxy-D$\left[1-{ }^{14} \mathrm{C}\right]$-glucose (PerkinElmer) 45 minutes before the end of clamp experiments. At the end of the experiments, mice were euthanized, and tissues were removed and immediately frozen in liquid nitrogen for analysis of 2 -deoxy-D- $\left[1-{ }^{14} \mathrm{C}\right]$-glucose content and insulin signaling. Tissue-specific glucose uptake was calculated as previously described (13). 
In vivo insulin signaling studies. In vivo insulin signaling experiments were performed on mice after an 8-hour lipid infusion as described above. Mice were injected with $10 \mathrm{U} / \mathrm{kg} \mathrm{BW}$ of human insulin (Lilly) or saline via the jugular vein. Five minutes after injection, skeletal muscle (gastrocnemius) was dissected and frozen in liquid nitrogen for immunoprecipitation and immunoblotting analysis of insulin signaling proteins.

Immunoprecipitation and immunoblotting. Skeletal muscle (gastrocnemius) from in vivo insulin signaling and hyperinsulinemic-euglycemic studies was homogenized in a modified radioimmunoprecipitation assay (RIPA) and supplemented with $1 \%$ protease inhibitor cocktail (SigmaAldrich) and 1\% tyrosine phosphatase inhibitor cocktail (Sigma-Aldrich). Immunoprecipitation and immunoblotting were conducted as previously described (21). Rabbit polyclonal anti-insulin receptor antibody was purchased from Santa Cruz Biotechnology Inc. Rabbit polyclonal anti-IRS-1, phospho-IRS-1 (Ser307), mouse monoclonal anti-phosphotyrosine 4G10, rabbit polyclonal anti-p85 antibodies, and protein A agarose were obtained from Upstate USA Inc.

EMSA. After lipid infusion, adipose tissue was collected for EMSA analysis. Adipose tissue nuclear extracts were prepared as previously described (44). The consensus NF- $\mathrm{KB}$ oligonucleotides (Promega) were end-labeled with $\left[\gamma^{32} \mathrm{P}\right]$ ATP (PerkinElmer) using T4 polynucleotide kinase (Promega). The protein-DNA complexes were resolved on a Novex 6\% DNA retardation gel (Invitrogen). A reaction containing 20 -fold excess of unlabeled competitor oligonucleotides (cold) was also included in each run. Gels were dried and analyzed by a PhosphorImager system (Molecular Dynamics).

ChIP assay. After lipid infusion, adipose tissue was collected for ChIP assay. ChIP assays were conducted using a ChIP assay kit (Upstate) according to the manufacturer's instructions, with a modification. Briefly, adipose tissue was cut into small pieces and fixed with $1 \%$ of formaldehyde. Samples were then homogenized in cell lysis buffer (5 mM PIPES, $85 \mathrm{mM} \mathrm{KCl}$, and $0.5 \% \mathrm{NP}-40$, supplemented with protease inhibitors, $\mathrm{pH}$ 8.0) using a dounce homogenizer to isolate nuclei. The nuclei were resuspended in nuclei lysis buffer $(50 \mathrm{mM}$ Tris- $\mathrm{HCl}, 10 \mathrm{mM}$ EDTA, and $1 \%$ SDS, supplemented with protease inhibitors, $\mathrm{pH} 8.1$ ) and sonicated to shear genomic DNA to an average fragment length of 200-1,000 bp. Lysates were centrifuged, and the supernatants were collected. Fifty microliters of each sample was removed as the input control. The supernatants underwent overnight immunoprecipitation, elution, reverse cross-linking, and protease $\mathrm{K}$ digestion, according to the manufacturer's manual. A mock immunoprecipitation without antibody was also included for each sample. The DNAs recovered from phenol/chloroform extraction were used for SYBR Green quantitative PCR (Applied Biosystems), and the DNA quantitation value of each sample was further normalized with the DNA quantitation of individual input control. The primer sequences for the NF- $\mathrm{KB}$ binding sites on the IL- 6 promoter were 5'-GACATGCTCAAGTGCTGAGTCAC-3' (forward) and 5'-AGATTGCACAATGTGACGTCG-3' (reverse), and the primer sequences for the MCP-1 promoter were 5'-CAGCATCTGGAGCTCACATTCC-3' (forward) and 5'GCATGAACAAGTTGAGAGATGCC-3' (reverse).

DIO studies. Six-week-old mice ( $n=8$ per group) were placed on either chow diet (6\% of the calories as fat; Teklad F6 Rodent Diet 8664; Harlan Teklad) or high-fat diet (59\% of the calories as fat; F3282; Bio-Serv). Body weight and food intake were measured weekly, if not otherwise indicated. At 26 weeks, mice were subjected to dual-energy x-ray absorptiometry to examine body composition (Lunar PIXImus mouse densitometer; PIXImus). Intraperitoneal insulin tolerance tests were performed at 36 weeks. Mice were injected with insulin (Humulin R; Lilly) after 4 hours of fasting, and glucose levels were measured at $0,15,30,60,90$, and 120 minutes using a OneTouch Ultra glucometer (LifeScan).

Statistics. All data are expressed as mean \pm SEM. Data were evaluated for statistical significance by 1 -way ANOVA, and statistical significance for comparison of means of different groups was calculated by the least-significant-difference test using the SPSS software package version 11.5. $P<0.05$ was considered significant.

\section{Acknowledgments}

This work was supported by NIH grant DK R37 28082 to J.S. Flier. We would like to thank members of the Flier lab for helpful discussions and assistance during the course of this study.

Received for publication April 24, 2006, and accepted in revised form September 12, 2006.

Address correspondence to: Jeffrey S. Flier, Beth Israel Deaconess Medical Center, 330 Brookline Avenue, Finard 202, Boston, Massachusetts 02215, USA. Phone: (617) 667-9050; Fax: (617) 667-9054; E-mail: jflier@bidmc.harvard.edu.
1. Kahn, B.B. and Flier, J.S. 2000. Obesity and insulin resistance. J. Clin. Invest. 106:473-481.

2. Wellen, K.E., and Hotamisligil, G.S.2003. Obesity-induced inflammatory changes in adipose tissue. J. Clin. Invest. 112:1785-1788. doi:10.1172/ JCI200320514.

3. Yamauchi, T., et al. 2001. The fat-derived hormone adiponectin reverses insulin resistance associated with both lipoatrophy and obesity. Nat. Med. 7:941-946.

4. Steppan, C.M., et al. 2001. The hormone resistin links obesity to diabetes. Nature. 409:307-312.

5. Yang, Q., et al. 2005. Serum retinol binding protein 4 contributes to insulin resistance in obesity and type 2 diabetes. Nature. 436:356-362.

6. Uysal, K.T., Wiesbrok., S.M., Marino, M.W., and Hotamisligil, G.S. 1997. Protection from obesityinduced insulin resistance in mice lacking TNF-alpha function. Nature. 389:610-614.

7. Wellen, K.E., and Hotamisligil, G.S. 2005. Inflammation, stress, and diabetes. J. Clin. Invest. 115:1111-1119. doi:10.1172/JCI200525102.

8. Lam, T.T., Van De Werve, G., and Giacca, A. 2003. Free fatty acids increase basal hepatic glucose production and induce hepatic insulin resistance at different sites. Am. J. Physiol. Endocrinol. Metab.
284:E281-E290.

9. Boden, G., et al. 2005. Free fatty acids produce insulin resistance and activate the proinflammatory nuclear factor- $\mathrm{\kappa B}$ pathway in rat liver. Diabetes. 54:3458-3465.

10. Dresner, A., et al. 1999. Effects of free fatty acids on glucose transport and IRS-1-associated phosphatidylinositol 3-kinase activity. J. Clin. Invest. 103:253-259

11. Kim, J.K., et al. 2004. PKC- $\theta$ knockout mice are protected from fat-induced insulin resistance. J. Clin. Invest. 114:823-827. doi:10.1172/JCI200422230.

12. Yuan, M., et al. 2001. Reversal of obesity- and dietinduced insulin resistance with salicylates or targeted disruption of IKK $\beta$. Science. 293:1673-1677.

13. Kim, J.K., et al. 2001. Prevention of fat-induced insulin resistance by salicylate. J. Clin. Invest. 108:437-446. doi:10.1172/JCI200111559.

14. Hirosumi, J., et al. 2002. A central role for JNK in obesity and insulin resistance. Nature. 420:333-336.

15. Medzhitov, R. 2001. Toll-like receptors and innate immunity. Nat. Rev. Immunol. 1:135-145.

16. Zuany-Amorim, C., Hastewell, J., and Walker, C. 2002. Toll-like receptors as potential therapeutic targets for multiple diseases. Nat. Rev. Drug Discov. 1:797-807.

17. Lee, J.Y., Sohn, K.H., Rhee, S.H., and Hwang, D.
2001. Saturated fatty acids, but not unsaturated fatty acids, induce the expression of cyclooygenase-2 mediated through Toll-like receptor 4. J. Biol. Chem. 276:16683-16689.

18. Lee, J.Y., et al. 2003. Reciprocal modulation of Toll-like receptor-4 signaling pathways involving MyD88 and phosphatidylinositol 3- kinase/AKT by saturated and polyunsaturated fatty acids. J. Biol. Chem. 278:37041-37051.

19. Hwang, D. 2001. Modulation of the expression of cyclooxygenase- 2 by fatty acids mediated through Toll-like receptor 4-derived signaling pathways. FASEB J. 15:2556-2564.

20. Cai, D., et al. 2005. Local and systemic insulin resistance resulting from hepatic activation of IKK- $\beta$ and NF-кB. Nat. Med. 11:183-190.

21. Arkan, M.C., et al. 2005. IKK- $\beta$ links inflammation to obesity-induced insulin resistance. Nat. Med. 11:191-198.

22. Weisberg, S.P., et al. 2003. Obesity is associated with macrophage accumulation in adipose tissue. J. Clin. Invest. 112:1796-1808. doi:10.1172/ JCI200319246.

23. Xu, H., et al. 2003. Chronic inflammation in fat plays a crucial role in the development of obesity-related insulin resistance. J. Clin. Invest. 112:1821-1830. 
doi:10.1172/JCI200319451.

24. Boden, G., et al. 1991. Effects of fat on insulin-stimulated carbohydrate metabolism in normal men. J. Clin. Invest. 88:960-966.

25. Boden, G. 2003. Effects of free fatty acids on glucose metabolism: significance for insulin resistance and type 2 diabetes. Exp. Clin. Endocrinol. Diabetes. 111:121-124.

26. Nguyen, M.T.A., et al. 2005. JNK and TNF- $\alpha$ mediate free fatty acid-induced insulin resistance in 3T3-L1 adipocytes. J. Biol. Chem. 280:35361-35371.

27. Gao, Z., et al. 2004. Inhibition of insulin sensitivity by free fatty acids requires activation of multiple serine kinases in 3T3-L1 adipocytes. Mol. Endocrinol. 18:2024-2034.

28. Ajuwon, K.M., and Spurlock, M.E. 2005. Palmitate activates the NF- $\mathrm{KB}$ transcription factor and induces IL- 6 and TNF- $\alpha$ expression in 3T3-L1 adipocytes. J. Nutr. 135:1841-1846.

29. Itani, S.I., Ruderman, N.B., Schmieder, F., and Boden, G. 2002. Lipid-induced insulin resistance in human muscle is associate with changes in diacylglycerol, protein kinase C, and IkappaB- $\alpha$. Diabetes. 51:2005-2011.

30. Sinha, S., Perdomo, G., Brown, N.F., and O'Doherty, R.M. 2004. Fatty acid-induced insulin resistance in L6 myotubes is prevented by inhibition of activation and nuclear localization of nuclear factor kappaB. J. Biol. Chem. 279:41294-41301.
31. Weigert, C., et al. 2004. Palmitate, but not unsaturated fatty acids, induces the expression of interleukin- 6 in human myotubes through proteasomedependent activation of nuclear factor-kappaB. J. Biol. Chem. 279:23942-23952.

32. Staiger, H., et al. 2004. Palmitate-induced interleukin-6 expression in human coronary artery endothelial cells. Diabetes. 53:3209-3216.

33. Van Epps-Fung, M., Williford, J., Wells, A., and Hardy, R.W. 1997. Fatty acid-induced insulin resistance in adipocytes. Endocrinology. 138:4338-4345.

34. Hirasawa, A., et al. 2005. Free fatty acids regulate gut incretin glucagon-like peptide-1 secretion through GPR120. Nat. Med. 11:90-94.

35. Itoh, Y., et al. 2003. Free fatty acids regulate insulin secretion from pancreatic beta cells through GPR40. Nature. 422:173-176.

36. Shi, H., Tzameli, I., Bjorbak, C., and Flier, J.S. 2004 Suppressor of cytokine signaling 3 is a physiological regulator of adipocyte insulin signaling. J. Biol. Chem. 279:34733-34740.

37. Frost, R.A., Nystrom, G.J., and Lang, C.H. 2004 Lipopolysaccharide stimulates nitric oxide synthase-2 expression in murine skeletal muscle and $\mathrm{C}(2) \mathrm{C}(12)$ myoblasts via Toll-like receptor-4 and c-Jun $\mathrm{NH}(2)$-terminal kinase pathways. Am. J. Physiol. Cell Physiol. 287:C1605-C1615.

38. Frost, R.A., Nystrom, G.J., and Lang, C.H. 2003. Lipopolysaccharide and proinflammatory cytokines stim- ulate interleukin- 6 expression in $\mathrm{C} 2 \mathrm{C} 12$ myoblasts: role of the Jun $\mathrm{NH}_{2}$-terminal kinase. Am. J. Physiol. Regul. Integr. Comp. Physiol. 285:R1153-R1164.

39. Kim, J.K., et al. 2004. Inactivation of fatty acid transport protein 1 prevents fat-induced insulin resistance in skeletal muscle. J. Clin. Invest. 113:756-769. doi:10.1172/JCI200418917.

40. Laflamme, N., and Rivest, S. 2001. Toll-like receptor 4: the missing link of the cerebral innate immune response triggered by circulating gramnegative bacterial cell wall components. FASEB J. 15:155-163.

41. Hoshino, K., et al. 1999. Cutting edge: Toll-like receptor 4 (TLR4)-deficient mice are hyporesponsive to lipopolysaccharide: evidence for TLR4 as the Lps gene product. J. Immunol. 162:3749-3752.

42. Malley, R., et al. 2003. Recognition of pneumolysin by Toll-like receptor 4 confers resistance to pneumococcal infection. Proc. Natl. Acad. Sci. U. S. A. 100:1966-1971.

43. Shi, H., Moustaid-Moussa, N., Wilkinson, W.O., and Zemel, M.B. 1999. Role of the sulfonylurea receptor in regulating adipocyte metabolism. FASEB J. 13:1833-1838.

44. Ajuwon, K.M., Jacobi, S.K., Kuske, J.L., and Spurlock, M.E. 2004. Interleukin-6 and interleukin-15 are selectively regulated by lipopolysaccharide and interferon-gamma in primary pig adipocytes. Am.J. Physiol. Regul. Integr. Comp. Physiol. 286:R547-R553. 